\title{
Untrennbar: Intensivmedizin und Beatmungsmedizin
}

\author{
Inseparable: Intensive Care Medicine and Mechanical Ventilation
}

Autoren

Institute
W. Windisch ${ }^{1}$, J. Geiseler ${ }^{2}$

1 Lungenklinik; Kliniken der Stadt Köln gGmbH, Universität Witten/Herdecke

${ }^{2}$ Klinik für Intensivmedizin, Schlaf- und Beatmungsmedizin, Asklepios Fachkliniken München-Gauting

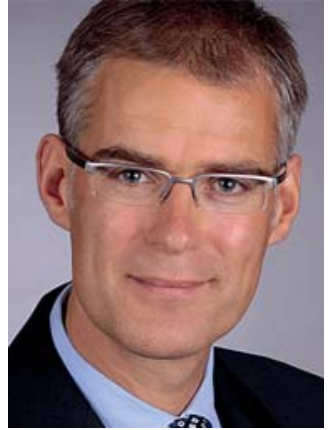

Prof. Dr. med. W. Windisch

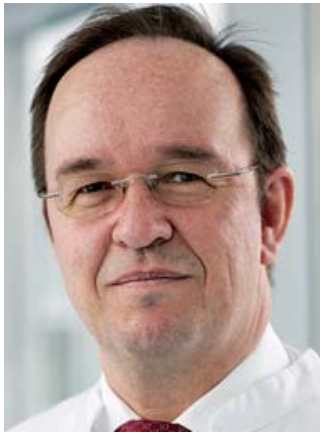

Dr. med. J. Geiseler

\section{Bibliografie}

DOI http://dx.doi.org/

10.1055/s-0034-1365313

Pneumologie 2014; 68: 171-172

(c) Georg Thieme Verlag KG

Stuttgart · New York

ISSN 0934-8387

Korrespondenzadresse

Prof. Dr. med.

Wolfram Windisch

Chefarzt Abt. Pneumologie Lungenklinik Merheim Kliniken der Stadt Köln gGmbH Ostmerheimer Straße 200 51109 Köln

windischw@kliniken-koeln.de
In kaum einem Bereich der modernen Medizin hat es in den letzten Jahrzehnten so viele Fortschritte wie in der Intensivmedizin gegeben [1]. Dies liegt zum einen an hochkomplexen technologischen Entwicklungen zur Behandlung des Ein-Organversagens (z.B. ECMO-Therapie); zum anderen sind es die Weiterentwicklungen in der Pharmakotherapie und die Verbesserungen des detaillierten Monitorings der Organfunktionen eines kritisch kranken Menschen [1].

Für diese und auch zukünftige Entwicklungen in der Intensivmedizin spielen die Behandlung von Patienten mit Atemversagen und insbesondere die Beatmungsmedizin die tragende Rolle. Fünf wesentliche Gründe sind hierfür verantwortlich:

1. In der historischen Entwicklung der modernen Intensivmedizin bildet die Etablierung von Systemen zur Behandlung der respiratorischen Insuffizienz den wesentlichen Eckpfeiler [2]. Die Entwicklung der „Eisernen Lunge“ im Jahre 1928 wurde gefolgt von der bedeutsamen Etablierung der invasiven Beatmung über ein Tracheostoma zur Behandlung der akuten respiratorischen Insuffizienz im Zuge der verheerenden Polioepidemie in den 50er Jahren des letzten Jahrhunderts [2]. Revolutionär waren im Weiteren die Einführung der nicht-invasiven Beatmung Ende der 80er Jahre sowie aktuell die Etablierung extrakorporaler Systeme zur Aufrechterhaltung einer suffizienten Oxygenierung, aber auch zur Elimination von Kohlendioxid.

2. Chronische Lungenerkrankungen, insbesondere die COPD, spielen bei Intensivpatienten eine tragende Rolle. So lag in einer großen österreichischen Studie die Prävalenz der COPD aller intensivmedizinisch betreuten Patienten global bei $9 \%$ [3]. Diese konnte zudem als Risikofaktor für ein prolongiertes Weaning und auch für eine erhöhte Mortalität identifiziert werden. Schließlich wurde ein Anstieg der Zahl von COPD-Patienten mit akuter respiratori- scher Insuffizienz um 2/3 innerhalb der letzten 11 Jahre dokumentiert - eine fundamentale epidemiologische Bedeutung dieser Erkrankung für die Intensivmedizin.

3. Ein prolongiertes Weaning und die Fortsetzung einer Beatmungstherapie als außerklinische Beatmung gewinnen rasant an Bedeutung. So ist bekannt, dass der eigentliche Weaningprozess ca. 40 bis $50 \%$ der Gesamtdauer einer mechanischen Ventilation einnimmt [4]. Durch die zunehmenden technischen Möglichkeiten, aber auch bedingt durch den demografischen Wandel, werden zunehmend Patienten im fortgeschrittenen Lebensalter mit zunehmender Anzahl und Schwere von Komorbiditäten intensivmedizinisch betreut. Hierdurch erhöht sich das Risiko eines prolongierten Weanings auch mit Gefahr des Weaningversagens, sodass die Intensivmedizin für diese Patienten ergänzt werden muss durch ein Programm einer Überleitung in eine außerklinische nichtinvasive oder invasive Beatmung $[4,5]$.

4. Entsprechend der zunehmenden Bedeutung der Langzeitbeatmung und der chronischen respiratorischen Insuffizienz ist eine Differenzierung in der Intensiv- und Beatmungstherapie auch unter organisatorischen Gesichtspunkten zu beobachten. Neben der High-EndIntensivstation haben sich zunehmend respiratorisch ausgerichtete Intermediate-Care-Stationen etabliert. Zudem werden zunehmend Weaning-Stationen/Weaning-Zentren aufgebaut, zusätzlich zur Ausweisung eigenständiger Bereiche zur Etablierung einer außerklinischen Beatmung $[1,4]$. Hier hat federführend die Deutsche Gesellschaft für Pneumologie und Beatmungsmedizin e.V. einen Zertifizierungsprozess für Weaning-Zentren (WeanNet) ins Leben gerufen und zudem Leitlinien zum prolongierten Weaning [4], zum Akuteinsatz der nicht-invasiven Beatmung [6] sowie zur außerklinischen Beatmung [5] etabliert. 
5. Im modernen Abrechnungssystem nach Fallpauschalen besitzen Beatmungs-DRGs einen hohen Erlösfaktor, allerdings auch hohe hinterlegte Kosten. Gleichzeitig fand zwischen 1991 und 2009 in Deutschland ein Abbau von ca. 135000 Krankenhausbetten statt [7]. Die Anzahl von Intensivbetten sowohl in kleinen als auch in großen Krankenhäusern hat allerdings entgegengesetzt zu dieser Entwicklung zugenommen, was auch schon vor Einführung des DRG-Abrechnungssystems zu beobachten war und damit nicht ausschließlich durch den Systemwechsel bedingt ist. Vielmehr sind der demografische Wandel, aber auch technische Neuentwicklungen, mit verantwortlich für eine Steigerung der Intensivkapazitäten [7]. Dies betrifft insbesondere Deutschland, welches im inter-europäischen Vergleich mit Abstand die höchsten Intensiv-Kapazitäten aufweist $[7,8]$. Grundsätzlich war zu befürchten gewesen, dass eine Steigerung der Intensivkapazitäten zu einer sinkenden Betten-Auslastung führen könnte, da durch das DRG-System der Druck erhöht wurde, Intensivkapazitäten sparsam zu nutzen; hier sind in den entsprechenden DRGs Intensivaufenthalte nur anteilig abgebildet. Die Abrechnung nach Fallpauschalen erlaubt aber keine direkte Abrechnung eines Behandlungstages auf der Intensivstation im Gegensatz zum früher gültigen System der Abrechnung nach Intensivpflegesätzen. Eine Ausnahme bilden hier die Beatmungs-DRGs, was die fundamentale ökonomische Bedeutung der Beatmungsmedizin innerhalb der Intensivmedizin unterstreicht. Entsprechend wird in den letzten 20 Jahren nicht nur eine Steigerung der Intensivkapazitäten, sondern zudem auch eine steigende Auslastung dieser gesteigerten Intensivkapazitäten von über $80 \%$ in den meisten Krankenhäusern beobachtet [7].

In Europa ist im Gegensatz zu den USA die führende Rolle des Fachgebiets Pneumologie in vielen Ländern für den Bereich der Intensivmedizin noch unterrepräsentiert [1]. Dies trifft auch für Deutschland zu. In naher Zukunft aber wird die Pneumologie eine zentrale Rolle im Bereich der Intensivmedizin spielen müssen. Dies ist insbesondere darin begründet, dass Beatmungsmedizin und Intensivmedizin in unserer heutigen Moderne nicht voneinander zu trennen sind. Die Physiologie und Pathophysiologie der Beatmung v.a. bei Atempumpenschwäche, aber auch bei Oxygenierungsstörungen, sind ureigene pneumologische Themenschwerpunkte. Wie dargestellt bilden die technischen Entwicklungen beim respiratorischen Versagen historische, aber auch zukünftige Meilensteine in der Entwicklung der Intensivmedizin. Zudem ist die Bedeutung von pneumologischen Erkrankungen und insbesondere der COPD für die Betreuung von inten- sivmedizinischen Patienten unbestreitbar. Auch der demografische Wandel mit älteren und komorbiden Patienten führt dazu, dass ein prolongiertes Weaning und die Einrichtung einer außerklinischen Beatmung von der eigentlichen Intensivmedizin nicht mehr abzutrennen sind. Gerade hier müssen auch End-of-LifeKonzepte entwickelt werden, für die insbesondere die Betrachtung der respiratorischen Situation durch den Pneumologen hinsichtlich der Prognose entscheidend ist. Schließlich ist die Beatmungsmedizin als wirtschaftlich tragender Faktor innerhalb der Intensivmedizin klar etabliert. Die wirtschaftlichen Anreize dürfen aber nicht dazu führen, die Beatmungs- und Intensivmedizin nach primär wirtschaftlichen Gesichtspunkten auszurichten. Es ist daher die Aufgabe der Verantwortlichen, gleichermaßen eine Qualitätssicherung in der Beatmungs- und Intensivmedizin sicherzustellen. Aufgrund des dargestellten fachlichen Bezugs kommt hier der Pneumologie eine Schlüsselrolle zu.

Vor diesen Hintergründen ist das Fachgebiet der Pneumologie aufgerufen, sich detailliert mit der Beatmungs- und Intensivmedizin zu beschäftigen. Daher werden in den folgenden Ausgaben der Fachzeitschrift Pneumologie Beiträge zur Serie „Intensivund Beatmungsmedizin“ publiziert werden.

\section{Literatur}

1 European Lung white book. Respiratory Intensive Care. http://www. erswhitebook.org/

2 Windisch $W$. Chronische respiratorische Insuffizienz: Rolle der außerklinischen Beatmung. Pneumologie 2010; 64: 600-603

3 Funk GC, Bauer P, Burghuber OC et al. Prevalence and prognosis of COPD in critically ill patients between 1998 and 2008. Eur Respir J 2013; 41: $792-799$

4 Schönhofer B, Geiseler J, Dellweg D et al. Prolongiertes Weaning. S2kLeitlinie herausgegeben von der Deutschen Gesellschaft für Pneumologie und Beatmungsmedizin e.V. Pneumologie 2014; 68: 19-75

5 Windisch W, Brambring J, Budweiser S et al. Nichtinvasive und invasive Beatmung als Therapie der chronischen respiratorischen Insuffizienz. S2-Leitlinie herausgegeben von der Deutschen Gesellschaft für Pneumologie und Beatmungsmedizin e.V. Pneumologie 2010; 64: 207-240

6 Schönhofer B, Kuhlen R, Neumann P et al. Nichtinvasive Beatmung als Therapie der akuten respiratorischen Insuffizienz - S3-Leitlinie herausgegeben von der Deutschen Gesellschaft für Pneumologie und Beatmungsmedizin. Pneumologie 2008; 62: 449-479

7 Thattil R, Klepzig D, Schuster M. Intensivkapazitäten in Deutschland: Vorhaltung und Nutzung zwischen 1991 and 2009. Anaesthesist 2012; 61: $56-62$

8 Rhodes A, Ferdinande P, Flaatten $H$ et al. The variability of critical care bed numbers in Europe. Intensive Care Med 2012; 38: 1647 - 1653 